Zhiquan Liu

https://doi.org/10.21278/TOF.43204

ISSN 1333-1124

eISSN 1849-1391

\title{
SHIP COURSE KEEPING USING DIFFERENT SLIDING MODE CONTROLLERS
}

\begin{abstract}
Summary
This study addresses three sliding mode heading controllers for dealing with uncertain wave disturbances. A nonlinear steering model is derived, and the feedback linearization method is chosen to simplify the nonlinear system in this study. The adaptive method and disturbance observer technique are proposed for course keeping and ensuring robust performance of the time varying wave moment and actuator dynamics. Finally, the simulation results on a navy ship illustrate the effectiveness of the presented control algorithms for course keeping.
\end{abstract}

Keywords: $\quad$ sliding mode control, course keeping, adaptive control, nonlinear disturbance observer

\section{Introduction}

A surface vessel moving in a seaway is always influenced by waves, especially in rough weather. Wave disturbances may cause cargo damage and can produce variations of motions. This is particularly true for fairways with heavy traffic and restricted area or depth of the water as well as for a ship on a safe course in the open sea. The ship autopilot is used to ensure safe sailing and to force the vessel track the path with a desired forward speed by using designed controllers. A detailed theoretical analysis of a proportional integral derivative (PID) controller for the ship autopilot was proposed in 1922 [1]. Ship steering systems are designed to perform course keeping (a fixed heading) and course changing (a new heading) manoeuvers. Typically, the purpose of course keeping is to achieve a minimum course deviation with the smallest rudder angle when the ship follows a certain rectilinear path. However, in the course changing manoeuver, it is desirable to follow another path with minimum rudder effort as quickly as possible.

The PID controller is the simplest controller which can deliver a good sailing performance in a fixed sea state. Fang et al. [2] developed a PD control system to damp the roll motion and keep the course in the seaway through rudder and fin actions. Banazadeth and Ghorbani [3] presented a PID heading controller with a novel system identification method using data analysis software. In regard to linear controllers, Saari and Djemai [4] developed a multi-structure internal model controller for the ship steering autopilot control according to 
different working points, and a model predictive controller was proposed for the steering and anti-roll control system [5]. A disturbance compensating predictive control scheme was developed for the ship heading control to satisfy the state constraints in waves [6]. In the literature, several kinds of non-linear controllers have been recently developed to overcome the nonlinear steering problem caused by wave disturbances. For instance, in the feedback linearization method, a typical state feedback linearization controller was selected for the ship course stabilization [7], an input-output linearization controller was developed for the steering control [8], and Zhang and Zhang presented a nonlinear feedback algorithm to improve the course keeping performance [9].

In this study, a nonlinear method that deals with unknown bounded external disturbances and guarantees robustness has been developed. Sliding mode controller (SMC) is a kind of strategy that satisfies the above requirements and can be implemented in the nonlinear ship autopilot system. The literature on the ship autopilot control using a sliding mode control algorithm has been reviewed as follows. Zhang et al. [10] addressed a path following sliding mode controller for a surface vessel in restricted waters. A decoupled SMC for the navigation of a supply ship was developed, and the parameters were optimized by a genetic algorithm (GA) [11]. Fang and Luo [12] proposed two different SMCs called "separate control" and "compact control" for the rudder roll stabilization and the heading control. Yu et al. [13] proposed a revised SMC law for an underactuated surface vessel by considering a first order sliding surface for the surge tracking problem and a second order sliding surface for yaw tracking errors. From a practical point of view, Ejaz and Chen [14] designed a SMC law which incorporated the actuator saturation for the autopilot system of a surface vessel. Harl and Balakrishnan [15] developed a robust second order SMC law for a modern warship to track a desired path. Perera and Soares [16] considered a pre-filtered SMC for the nonlinear steering problem with known bounded disturbances. Liu et al. [17] presented an adaptive SMC for the underactuated ship straight line tracking with parameter uncertainties. Aiming at developing the heading control for underactuated ships, Li et al. [18] proposed an active disturbance rejection control method to damp the effect of external disturbances. Liu et al. [19] introduced an adaptive hierarchical SMC to cope with the underactuated vessel trajectory tracking problem. Under sensorless condition, a high gain observer based SMC was developed to force an underactuated vessel to follow a predefined course [20] and a sliding mode backstepping control law was employed for the path following problem of underactuated surface vessels by means of parameter estimation [21]. In regard to autonomous underwater vehicles (AUV) tracking control, Zhang et al. [22] developed an adaptive second order SMC for the path following control of a portable AUV, and Elmokadem et al. [23] designed a terminal sliding mode control (TSMC) for the trajectory tracking of AUVs.

This study is limited to the problem of the nonlinear surface vessel course keeping with the assumption that the forward speed is constant. Several forms of sliding mode controllers are proposed to deal with unknown bounded external disturbances.

\section{Mathematical model}

Assuming a surface underactuated vessel moving forward with a constant speed $U$, the mathematical model for the ship motion in this study was introduced by Perez [24], and the dynamic equations are given as follows:

$$
\begin{aligned}
& I_{z z} \dot{r}+m x_{G} U r=N_{h y d}+N_{c}+d \\
& \dot{\psi}=r
\end{aligned}
$$


where the ship mass is $m$, the inertia in yaw is $I_{z z}$, the coordinate of the centre of gravity with respect to the body fixed frame is $x_{G}$. Yaw and the yaw rate are $\psi$ and $r$. The hydrodynamic force is denoted by $N_{h y d}$, and the control force (i.e. rudder moment) is denoted by $N_{c} . d=d(t)$ is the yaw moment caused by waves.

The hydrodynamic force equation of the ship is shown as follows:

$$
N_{\text {hyd }}=N_{\dot{r}} \dot{r}+N_{|U| r}|U| r+N_{r|r| r} r|r|
$$

The rudder is a device used for heading control in this investigation. The single rudder induced forces and moments can be expressed as follows:

$$
N_{c}=-\frac{1}{2} \rho A_{R} C_{L} U^{2} L C G
$$

where $\rho$ is the water density, $A_{R}$ is the area of the single rudder, $C_{L}=\left.\frac{\partial C_{L}}{\partial \delta}\right|_{\delta=0} \delta$ is the lift coefficient (i.e. the effect of the stall angle is not considered in this expression, it will be solved by limiting the rudder angle), $\delta=\delta(t)$ is the rudder angle, and $L C G$ is the distance from the centre of gravity to the rudder stock.

By combining the rudder action $N_{c}=N_{\delta} \delta$ and the wave moment d, the heading model is treated as:

$$
\left(I_{z z}-N_{\dot{r}}\right) \dot{r}=N_{|U| r}|U| r+N_{r|r|} r|r|-m x_{G} U r+N_{\delta} \delta+d
$$

where $N_{\delta}=-\left.\frac{1}{2} L C G \rho A_{R} U^{2} \frac{\partial C_{L}}{\partial \delta}\right|_{\delta=0}$.

This nonlinear model can be modified as

$$
\dot{r}=a_{1} r+a_{2} r|r|+a_{3} \delta+a_{4} d
$$

where $a_{1}=\frac{N_{|U| r}|U|-m x_{G} U r}{I_{z z}-N_{\dot{r}}}, a_{2}=\frac{N_{r|r|}}{I_{z z}-N_{\dot{r}}}, a_{3}=\frac{N_{\delta}}{I_{z z}-N_{\dot{r}}}, a_{4}=\frac{1}{I_{z z}-N_{\dot{r}}}$.

Assumption 1. The yaw $\psi$ and the yaw rate $r$ are measured accurately (i.e. without noise) by a gyroscopic compass and a yaw rate gyroscope, respectively.

Assumption 2. The disturbance (i.e. yaw moment) signal satisfies $|d(t)| \leq d_{\max }$, and $d_{\max }$ is an unknown positive constant.

Remark 1. In general, in sailing vessels six-degree-of-freedom (6-DOF) motions can be induced by the first order wave forces. Since we only consider the heading control, the general 6-DOF ship model can be reduced to the motion in yaw under the assumption that heave, roll, pitch, sway and surge modes induced by waves can be neglected.

\section{Control system}

The diagram of the heading control system is given in Fig. 1, where $\psi_{d}$ is the desired yaw angle and the yaw rate is set to zero. The diagram of the steering machine is shown in Fig. 2, which presents the relationship between the commanded rudder angle $\delta_{c}$ and the presented rudder angle $\delta$. 


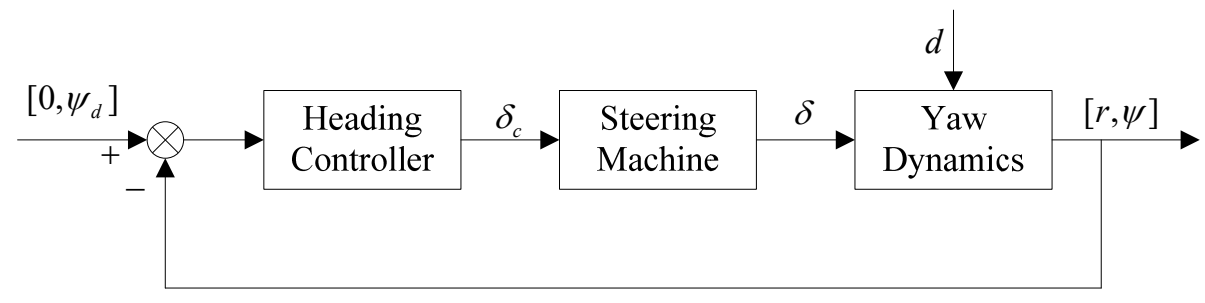

Fig. 1 Autopilot control system

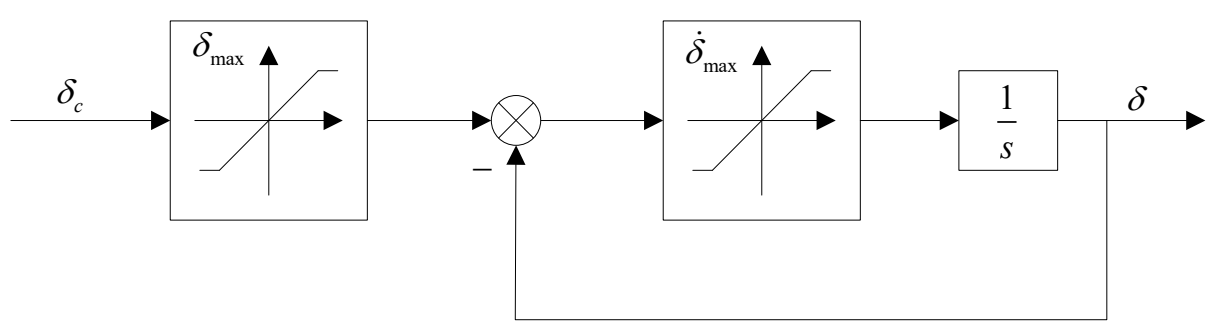

Fig. 2 Steering machine model

In this section, we present three types of sliding mode controllers used to reject the unknown bounded wave moment, which can maintain the system robustness. The following parts show the design process of the three controllers.

\subsection{Sliding mode controller}

In order to convert the nonlinear term in Eq. (6), firstly, the feedback linearization method is adopted. Define a new variable $u$ and the rudder control order is given as follows:

$$
\delta_{c}=\frac{1}{a_{3}}\left(u-a_{2} r|r|\right)
$$

Then the nonlinear steering system and its state space model are modified as follows:

$$
\begin{gathered}
\dot{r}=a_{1} r+u+a_{4} d \\
\dot{\mathbf{x}}=\mathbf{A} \mathbf{x}+\mathbf{B} u+\bar{d}
\end{gathered}
$$

where $\mathbf{x}=\left[\begin{array}{l}r \\ \psi\end{array}\right], \mathbf{A}=\left[\begin{array}{cc}a_{1} & 0 \\ 1 & 0\end{array}\right], \mathbf{B}=\left[\begin{array}{l}1 \\ 0\end{array}\right], \bar{d}=a_{4} d$.

By choosing a suitable control law, a sliding manifold is selected to obtain a relationship sufficient for each of the control element. Let the reference state be $\mathbf{x}_{\mathrm{d}}=\left[0, \psi_{\mathrm{d}}\right]$ and $\dot{\mathbf{x}}_{\mathrm{d}}=0$, the sliding manifold is defined as:

$$
s=\mathbf{h}^{\mathrm{T}} \mathbf{x}_{\mathrm{e}}=\mathbf{h}^{\mathrm{T}}\left(\mathbf{x}-\mathbf{x}_{\mathrm{d}}\right)
$$

where $\mathbf{h}=\left[h_{1}, h_{2}\right]^{\mathrm{T}}$ is a right eigenvector of $\mathbf{A}_{\mathrm{c}}\left(\right.$ i.e. $\left.\mathbf{A}_{\mathrm{c}}^{\mathrm{T}} \mathbf{h}=\lambda \mathbf{h}\right)$, and the weighting vector $\mathbf{h}$ is selected according to $\mathbf{A}_{\mathrm{c}}^{\mathrm{T}} \mathbf{h}=0$ for $\lambda=0$ [25].

In the SMC system, the defined control signal is written with a linear part (i.e. a state feedback controller) and a nonlinear part (i.e. a nonlinear switching controller),

$$
u=-\mathbf{k x}+u_{0}
$$

Substituting Eq. (11) into Eq. (9) we obtain

$$
\dot{\mathbf{x}}=\mathbf{A}_{\mathrm{c}} \mathbf{x}+\mathbf{B} u_{0}+\bar{d}
$$


where $\mathbf{A}_{\mathrm{c}}=\mathbf{A}-\mathbf{B} \mathbf{k}^{\mathrm{T}}$, and the feedback gain vector is defined as $\mathbf{k}=[k, 0]^{\mathrm{T}}$. follows:

The nonlinear controller which is used to reject uncertain wave moment is given as

$$
u_{0}=-\left(\mathbf{h}^{\mathrm{T}} \mathbf{B}\right)^{-1}\left[\mathbf{h}^{\mathrm{T}} \hat{\bar{d}}+\eta \operatorname{sgn}(s)\right]
$$

where $\hat{\bar{d}}$ is the estimate of $\bar{d}$.

Differentiating the sliding surface, then

$$
\dot{s}=-\eta \operatorname{sgn}(s)+\mathbf{h}^{\mathrm{T}} \Delta \bar{d}
$$

where $\lambda \mathbf{x}^{\mathrm{T}} \mathbf{h}=0$ if $\mathbf{h}$ is a right eigenvector, and $\mathbf{h}^{\mathrm{T}} \dot{\mathbf{x}}_{\mathrm{d}}=0$, and the estimation error is $\Delta \bar{d}=\bar{d}-\hat{\bar{d}}$.

As the wave moment is uncertain, a better guess for it is $\hat{\bar{d}}=0$. Then, the switching controller is

$$
u_{0}=-\left(\mathbf{h}^{\mathrm{T}} \mathbf{B}\right)^{-1} \eta \operatorname{sgn}(s)
$$

where $\eta>a_{4} d_{\max }\|\mathbf{h}\|=\bar{d}_{\max }\|\mathbf{h}\|$.

If the Lyapunov function is selected

$$
V=\frac{1}{2} s^{2}
$$

then

$$
\dot{V}=s \dot{s}=-\eta \operatorname{sgn}(s) s+s \mathbf{h}^{\mathrm{T}} \bar{d}=-\eta|s|+s \mathbf{h}^{\mathrm{T}} \bar{d} \leq 0
$$

In order to attenuate the chattering effect, the tanh function is adopted instead of the signum function. Hence, the total control law of the rudder angle is

$$
\delta_{c}=\frac{1}{a_{3}}\left[-k r-\frac{1}{h_{1}} \eta \tanh (s / \phi)-a_{2} r|r|\right]
$$

where $\phi$ is the boundary layer thickness.

\subsection{Adaptive sliding mode controller}

The SMC may need shorter time to reach the sliding manifold with a larger switching gain $\eta$, but the upper bound of the wave moment is difficult to obtain. In this section, a modified controller called adaptive sliding mode controller (ASMC) is presented, and the purpose of this method is to tune the switching gain without the knowledge of the external moment.

Considering $\hat{\bar{d}}=0$, the robust switching controller is modified as

$$
u_{0}=-\left(\mathbf{h}^{\mathrm{T}} \mathbf{B}\right)^{-1} \hat{\eta} \operatorname{sgn}(s)
$$

where $\hat{\eta}>0$ is the estimate of $\eta$.

The adaptation law for the tuning of the gain is

$$
\dot{\hat{\eta}}=\frac{1}{\alpha}|s|
$$

where $\alpha>0$ is the adaptation gain. A good value can also effectively avoid the exorbitant control activity and it is a positive constant in this study. 
Considering the same sliding surface function as in the above section, its differentiation can be shown as

$$
\dot{s}=-\hat{\eta} \operatorname{sgn}(s)+\mathbf{h}^{\mathrm{T}} \bar{d}
$$

In order to investigate whether this adaptive strategy can guarantee that the state trajectories reach the sliding manifold in finite time, a Lyapunov function is defined as

$$
V=\frac{1}{2} s^{2}+\frac{1}{2} \alpha \tilde{\eta}^{2}
$$

where $\tilde{\eta}=\hat{\eta}-\eta$ is the estimation error.

$$
\begin{aligned}
\dot{V} & =\frac{1}{2} s \dot{s}+\frac{1}{2} \alpha \tilde{\eta}^{2}=s\left[\mathbf{h}^{\mathrm{T}} \bar{d}-\hat{\eta} \operatorname{sgn}(s)\right]+\alpha(\hat{\eta}-\eta) \dot{\hat{\eta}} \\
& =s \mathbf{h}^{\mathrm{T}} \bar{d}-\hat{\eta}|s|+(\hat{\eta}-\eta)|s|=s \mathbf{h}^{\mathrm{T}} \bar{d}-\eta|s| \leq 0
\end{aligned}
$$

Selecting the tanh function as the switching function, the autopilot control order is

$$
\delta_{c}=\frac{1}{a_{3}}\left[-k r-\frac{1}{h_{1}} \hat{\eta} \tanh (s / \phi)-a_{2} r|r|\right]
$$

\subsection{Nonlinear disturbance observer based controller}

In the previous two sections, the estimate of the wave moment is treated as zero. An alternative method called disturbance observer (DO) is adopted here to deal with the issue of unknown disturbances. Since the yaw acceleration signal is not easy to get, a modified observer called nonlinear disturbance observer (NDO) is adopted here to estimate the external disturbance with the yaw and yaw rate signal [26, 27].

A new virtual variable with a function is defined as

$$
z=\hat{d}-p(r, \psi)
$$

where $\frac{\mathrm{d} p}{\mathrm{~d} t}=\frac{L(r, \psi)}{a_{4}} \dot{r}$.

Based on the formulation of the linear observer, the DO is written as follows:

$$
\begin{aligned}
\dot{\hat{d}} & =L(r, \psi) \tilde{d} \\
& =-L(r, \psi) z+L(r, \psi)\left[\frac{1}{a_{4}} \dot{r}-\frac{a_{1}}{a_{4}} r-\frac{a_{2}}{a_{4}} r|r|-\frac{a_{3}}{a_{4}} \delta-p(r, \psi)\right]
\end{aligned}
$$

where $\tilde{d}=d-\hat{d}$ is the observer error.

In general, the external wave moment varies more slowly than the observer dynamics. Therefore, it is reasonable to say that

$$
\dot{d}=0
$$

and

$$
\dot{\tilde{d}}=\dot{d}-\dot{\hat{d}}=-\dot{\hat{d}}=-L(r, \psi) \tilde{d}
$$


Define a positive constant a and let $L(r, \psi)=a$, then

$$
\begin{aligned}
& p(r, \psi)=\frac{a}{a_{4}} r \\
& \frac{\mathrm{d} p}{\mathrm{~d} t}=\frac{a}{a_{4}} \dot{r}
\end{aligned}
$$

Based on the former equations, the update law is treated as

$$
\dot{z}=\dot{\hat{d}}-\frac{\mathrm{d} p}{\mathrm{~d} t}=\dot{\hat{d}}-\frac{a}{a_{4}} \dot{r}=-a z+a\left[-\frac{a_{1}+a}{a_{4}} r-\frac{a_{2}}{a_{4}} r|r|-\frac{a_{3}}{a_{4}} \delta\right]
$$

Finally, the rudder command signal is given as follows:

$$
\delta_{c}=\frac{1}{a_{3}}\left[-k r-a_{4} \hat{d}-\frac{1}{h_{1}} \eta_{0} \tanh (s / \phi)-a_{2} r|r|\right]
$$

where $\eta_{0}>\|\mathbf{h}\| \cdot\|\Delta \bar{d}\|$ and $\hat{\bar{d}}=a_{4} \hat{d}$.

\section{Simulation results and discussion}

The proposed course keeping simulation is demonstrated and the principal particulars of the vessel with two rudders are listed in Table 1. The wave environment is simulated with a significant wave height of $1.5 \mathrm{~m}$ and an average period of $7.5 \mathrm{~s}$. In order to avoid the effect of stall angle, $\delta_{\max }=\delta_{\text {stall }}=25 \mathrm{deg}$, the vessel is equipped with two rudders, $\delta_{\min }=0.5 \mathrm{deg}$ and $\dot{\delta}_{\text {max }}=15 \mathrm{deg} / \mathrm{s}$. The initial values are $\mathbf{x}=(0,0)^{\mathrm{T}}, \eta=2, \eta_{0}=1.5$ and $\hat{\eta}(0)=1$. The controller parameters are set as follows: $\phi=1, k=0.1$ and $\left(\eta_{\min }, \eta_{\max }\right)=(1,10)$. The NDO gain is $a=23$ and the sailing encounter angle is $\beta=135^{\circ}$, and the time domain simulation is done by the fourth order Runge-Kutta numerical integration method with the time step of $0.1 \mathrm{~s}$.

Table 1 Principal particulars of the vessel

\begin{tabular}{|l|l|l|}
\hline Hull data & Value & Unit \\
\hline Length between perpendiculars (LPP) & 51.5 & $\mathrm{~m}$ \\
\hline Beam overall (B) & 8.6 & $\mathrm{~m}$ \\
\hline Draft at LPP/2 & 2.3 & $\mathrm{~m}$ \\
\hline Longitudinal center of gravity (LCG) & 20.4 & $\mathrm{~m}$ \\
\hline Displacement & 357.0 & $\mathrm{~m}^{3}$ \\
\hline Nominal ship speed (U) & 15 & $\mathrm{knots}$ \\
\hline Water density $(\rho)$ & 1025 & $\mathrm{~kg} / \mathrm{m}^{3}$ \\
\hline Gravity constant $(\mathrm{g})$ & 9.81 & $\mathrm{~m} / \mathrm{s}^{2}$ \\
\hline Rudder data (single) & Value & Unit \\
\hline Area $\left(A_{R}\right)$ & 1.5 & $\mathrm{~m}$ \\
\hline Span $(\mathrm{sp})$ & 1.5 & $\mathrm{~m}$ \\
\hline Mean cord $(\bar{c})$ & 1 & $\mathrm{~m}$ \\
\hline Stall angle $\left(\delta_{\text {stall }}\right)$ & 25 & $\mathrm{degree}$ \\
\hline Lift coefficient $\left(\partial C_{L} /\left.\partial \delta\right|_{\delta=0}\right)$ & 0.054 & \\
\hline
\end{tabular}


Table 2 Cost values

\begin{tabular}{|c|c|c|c|c|}
\hline $\begin{array}{c}\text { Performance } \\
\text { (RMSE) }\end{array}$ & SMCNDO & ASMC & SMC & Unit \\
\hline Yaw angle & 0.44 & 0.60 & 0.64 & degree \\
\hline Yaw rate & 0.39 & 0.48 & 0.48 & degree $/ \mathrm{s}$ \\
\hline Rudder angle & 8.89 & 9.12 & 8.67 & degree \\
\hline Rudder rate & 7.66 & 8.51 & 7.66 & degree $/ \mathrm{s}$ \\
\hline Wave moment & 713313.61 & 713313.61 & 713313.61 & $\mathrm{~N} \cdot \mathrm{m}$ \\
\hline NDO & 699968.28 & & & $\mathrm{~N} \cdot \mathrm{m}$ \\
\hline
\end{tabular}

Under the heading condition of $\beta=135^{\circ}$, the computation simulations of the heading control are presented in Figs. 3-8, and the corresponding simulation values (RMSE) are presented in Table 2. Fig. 3 shows the time traces of the wave height and the wave moment, the RMSE values of the wave moment and the output of NDO are almost equal, a better estimate performance can be obtained when a higher value of $L(r, \psi)$ is chosen, since a too high value may cause an algebraic loop in the simulation process, and the value $a=23$ chosen in this study is reasonable. Figs. 4 and 5 show time series of the yaw and the rudder angle, the ASMC can get a better course keeping performance with its smaller adaptation gain in comparison with the SMC because a larger switching gain $\eta$ corresponds to a shorter time to reach $s=0$, and the system robustness against the environmental disturbance is proven. The SMCNDO makes the smallest yaw and rudder responses, which is mainly due to the accurate estimation of the external disturbances.

(a) Wave height $(\mathrm{m})$
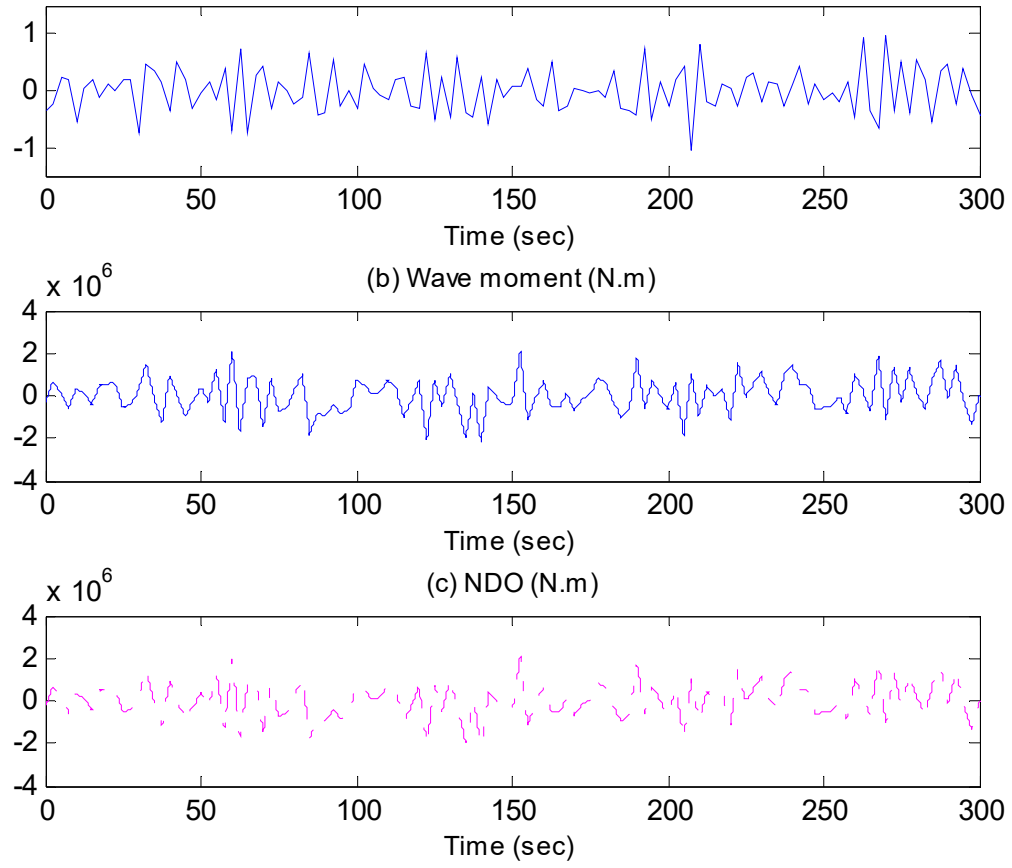

Fig. 3 Simulation of results of wave disturbance 


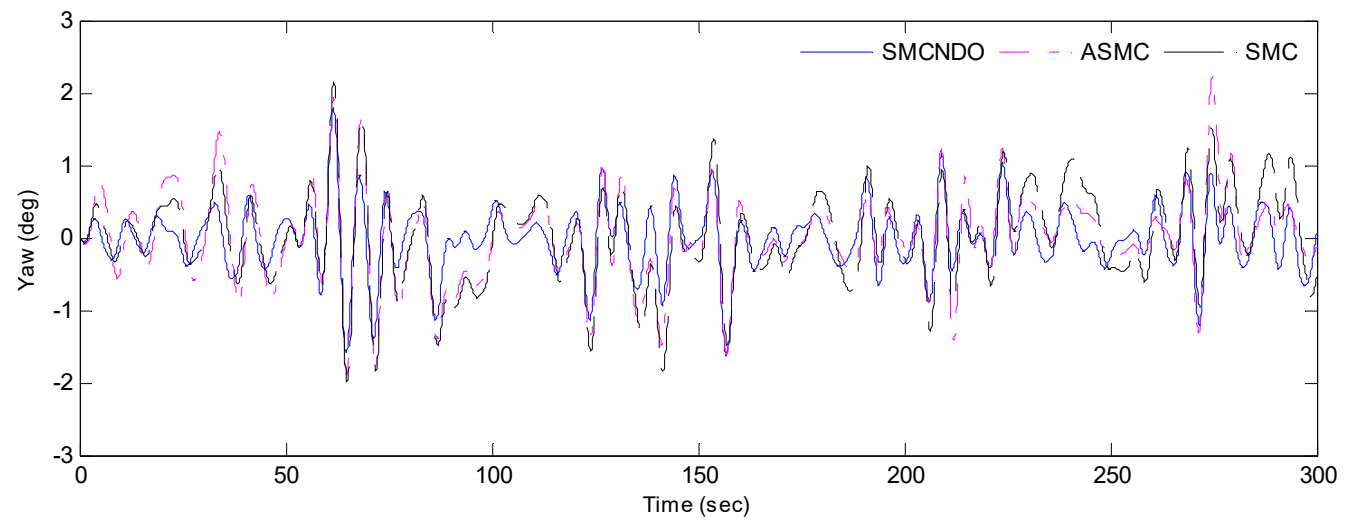

Fig. 4 Comparison of yaw angle responses

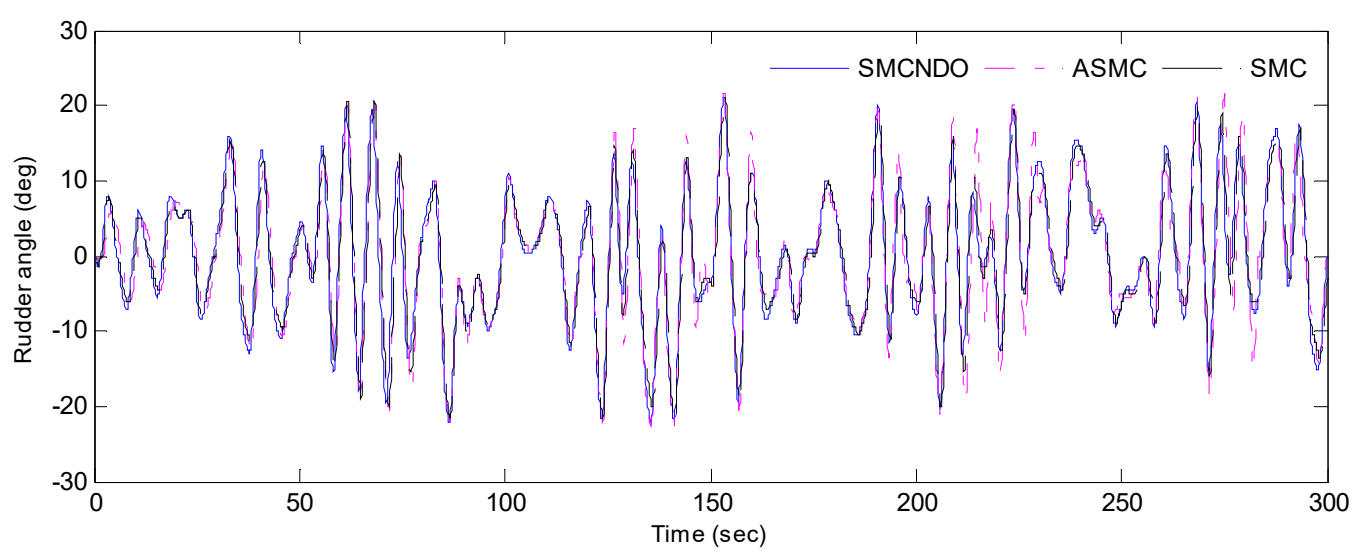

Fig. 5 Comparison of rudder angle responses

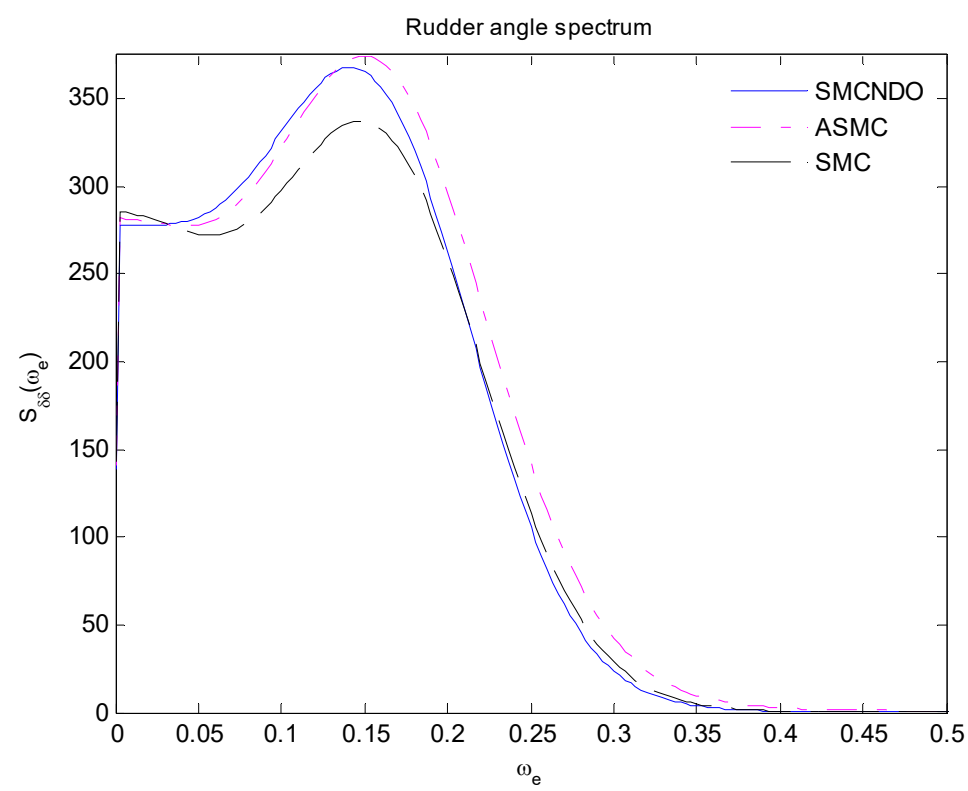

Fig. 6 Comparison of rudder angle spectrum 


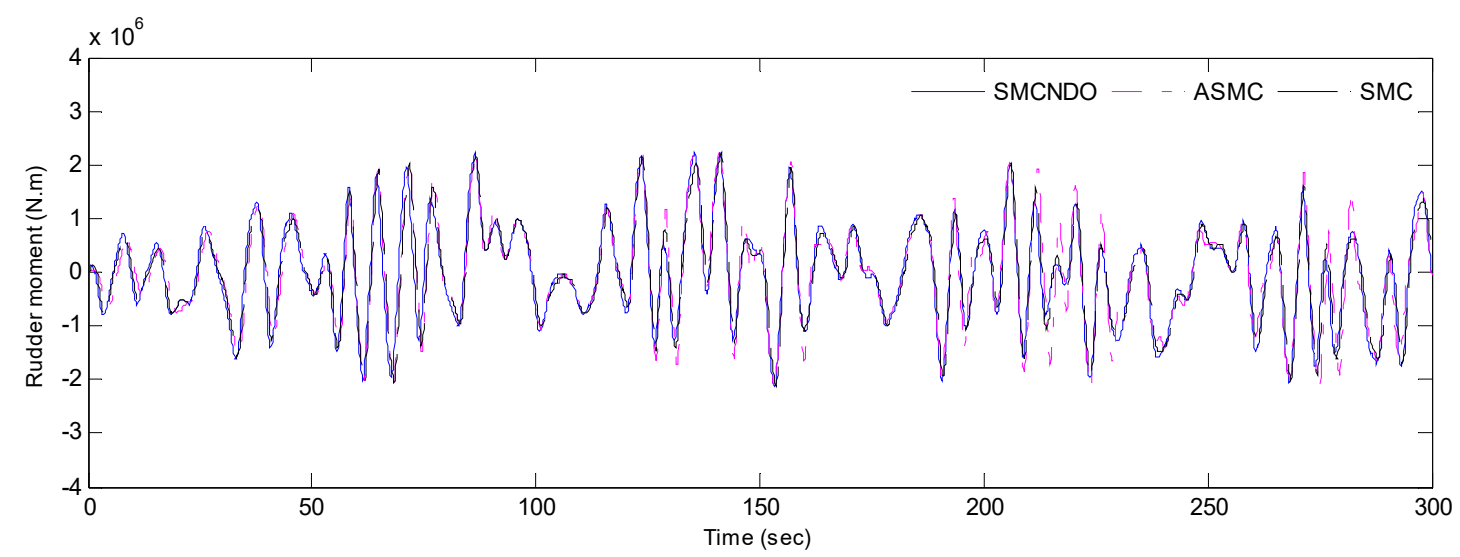

Fig. 7 Comparison of rudder moment

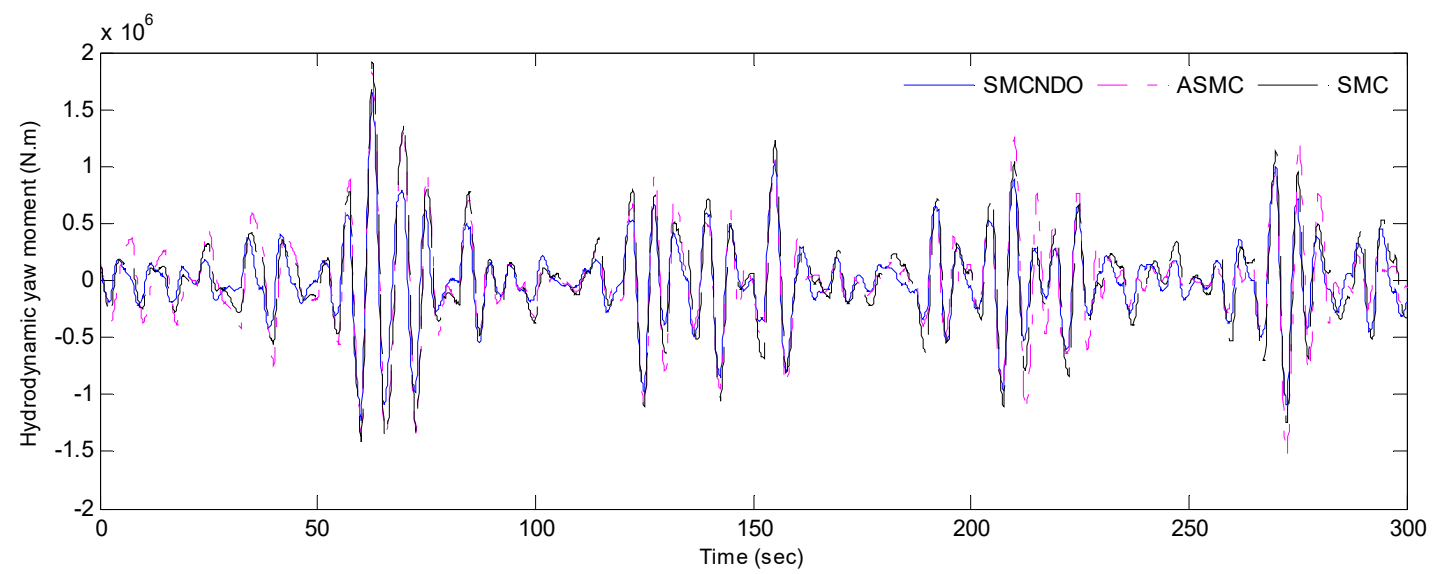

Fig. 8 Comparison of hydrodynamic yaw moment

In order to analyse the rudder angle responses further, the corresponding power spectrum densities are presented in Fig. 6. It shows that the ASMC may increase the energy consumption of the rudder servo system. The ASMC can achieve a good heading control performance based on the higher rudder angle cost value which increases the wear and tear of the steering machine. Since the rudder angle and the rudder rate are both constrained, the SMCNDO produces realistic conditions in the ship heading control performance. Considering the control laws (27) and (32), the switching gain $\eta_{0}$ is easier to determine than $\eta$, because $\eta_{0}$ depends on the upper bound on the estimation error (i.e. the deviation between the estimated value of the nonlinear observer and the actual value of the wave moment) of the disturbance and $\eta$ depends on the unknown upper bound on the wave moment. The SMC may get better results by higher gains and more actuator responses which should not be supported in practice. Figs. 7 and 8 present the corresponding time series of the yaw moment induced by the rudder and the hydrodynamic yaw moment, according to Eqs. (3) and (4), and the rudder moment that rejects the yaw moment induced by waves is determined by the rudder angle and the hydrodynamic moment is determined by the yaw rate.

\section{Conclusions}

This study presents three kinds of sliding mode controllers whose purpose is to deal with the unknown bounded wave moment in the nonlinear ship heading control problem. The parameters of the proposed controllers were set as the way in Ref. [27] and the constraint condition problem of the steering machine was solved. As shown in the simulation results, all controllers have successfully changed the ship actual course into the reference course. As 
noted in the simulations, the actuator responses approach the limitation because of the rudder order. The steering performance is evaluated with a trade-off between the actuator responses and the accuracy of the heading control. Comparing the results of the steering system, the yaw motion responses deliver the superior performance when an SMC with an NDO is applied. In this case, the actuator costs are lower and the course keeping results are better. In this study, a nonlinear yaw model is selected and the forward speed is assumed to be constant, while the heading control problem can also use a 3-DOF (i.e. surge, sway and yaw) model. Furthermore, speed is the time variant in practice because it can be reduced by added resistance. So, the 3-DOF uncertain nonlinear coupling ship model and the speed loss problem in the heading control system will be considered in the future work.

\section{REFERENCES}

[1] Fossen, T. I. 2011, Handbook of Marine Craft Hydrodynamics and Motion Control, West Sussex: Wiley.

[2] Fang, M. C., Lin, Y. H., and Wang, B. J. 2012, Applying the PD controller on the roll reduction and track keeping for the ship advancing in waves. Ocean Engineering, 54(4), 13-25. https://doi.org/10.1016/j.oceaneng.2012.07.006

[3] Banazadeh, A. and Ghorbani, M. T. 2013, Frequency domain identification of Nomoto model to facilitate kalman filter estimation and PID heading control of a patrol vessel. Ocean Engineering, 72(4), 344-355. https://doi.org/10.1016/j.oceaneng.2013.07.003

[4] Saari, H. and Djemai, M. 2012, Ship motion control using multi-controller structure. Ocean Engineering, 55(4), 184-190. https://doi.org/10.1016/j.oceaneng.2012.07.028

[5] Liu, C., Sun, J. and Zou, Z. 2015, Integrated line of sight and model predictive control for path following and roll motion control using rudder. Journal of Ship Research, 59(2), 99-112. https://doi.org/10.5957/JOSR.59.2.140057

[6] Li, Z. and Sun, J. 2012, Disturbance compensating model predictive control with application to ship heading control. IEEE Transactions on Control Systems Technology, 20(1), 257-265. https://doi.org/10.1109/TCST.2011.2106212

[7] Borkowski, P. 2014, Ship course stabilization by feedback linearization with adaptive object model, Polish Maritime Research. 21(81), 14-19. https://doi.org/10.2478/pomr-2014-0003

[8] Perera, L. P. and Soares, C. G. 2013, Lyapunov and Hurwitz based controls for input output linearization applied to nonlinear vessel steering. Ocean Engineering, 66, 58-68. https://doi.org/10.1016/j.oceaneng.2013.04.002

[9] Zhang, X. and Zhang, G. 2016, Design of a ship course keeping autopilot using a sine function based nonlinear feedback technique. The Journal of Navigation, 69(2), 246-256. https://doi.org/10.1017/S0373463315000612

[10] Zhang, R., Chen, Y. and Sun, Z. 2000, Path control of a surface ship in restricted waters using sliding mode. IEEE Transactions on Control System Technology, 80(4), 722-732. https://doi.org/10.1109/87.852916

[11] Alfardo-Cid, E., McGookin, E. W., Murray-Smith, D. J. and Fossen, T. I. 2005, Genetic algorithms optimization of decoupled sliding mode controllers: simulated and real results. Control Engineering Practice, 13(6), 739-748. https://doi.org/10.1016/j.conengprac.2004.07.004

[12] Fang, M. C. and Luo, J. H. 2007, On the tracking and roll reduction of the ship in random waves using different sliding mode controllers. Ocean Engineering, 34(3), 479-488. https://doi.org/10.1016/j.oceaneng.2006.03.004

[13] Yu, R., Zhu, Q., Xia, G. and Liu, Z. 2012, Sliding mode tracking control of an underactuated surface vessel. IET Control Theory and Applications, 6(3), 461-466. https://doi.org/10.1049/iet-cta.2011.0176

[14] Ejaz, M. and Chen, M. 2017, Sliding mode control design of a ship steering autopilot with input saturation. International Journal of Advanced Robotics System, 14(3), 1-13. https://doi.org/10.1177/1729881417703568

[15] Harl, N. and Balakrishnan, S. N. 2012, Impact time angle guidance with sliding mode control. IEEE Transactions on Control System Technology, 20(6), 1436-1449. https://doi.org/10.1109/TCST.2011.2169795

[16] Perera, L. and Soares, C. G. 2012, Pre-filtered sliding mode control for nonlinear ship steering associated with disturbances. Ocean Engineering, 51(3), 49-62. https://doi.org/10.1016/j.oceaneng.2012.04.014 
[17] Liu, C. Zou, Z. and Li, T. 2013, Adaptive robust sliding mode control for ship straight line tracking in random waves. Journal of Shanghai Jiaotong University (Science), 18(5), 549-553.

https://doi.org/10.1007/s12204-013-1430-1

[18] Li, R., Li, T., Bu, R., Zheng, Q. and Philip Chen, C. L. 2013, Active disturbance rejection with sliding mode control based course and path following for underactuated ships. Mathematical Problem in Engineering, 2013(1), 1-9. https://doi.org/10.1155/2013/743716

[19] Liu, C., Zou, Z. and Hou, X. 2015, Stabilization and tracking of underactuated surface vessels in random waves with fin based on adaptive hierarchical sliding mode control technique. Asian Journal of Control, 16(5), 1492-1500. https://doi.org/10.1002/asjc.920

[20] Qin, z., Lin, Z., Sun, H. and Yang, D. 2016, Sliding mode control of path following for underactuated ships based on high gain observer. Journal of Central South University, 23(12), 3356-3364. https://doi.org/10.1007/s11771-016-3401-9

[21] Sun, Z., Zhang, G., Yang, J. and Zhang, W. 2017, Research on sliding mode control for underactuated surface vessels via parameter estimation. Nonlinear Dynamics, 91(2), 1163-1175. https://doi.org/10.1007/s11071-017-3937-8

[22] Zhang, G., Huang, H., Qin, H., Li, Y., Gao, J. and Su, Y. 2018, A novel adaptive second order sliding mode path following control for a portable AUV. Ocean Engineering, 151, 82-92. https://doi.org/10.1016/j.oceaneng.2017.12.054

[23] Elmokadem, T., Zribi, M. and Youcef-Toumi, K. 2017, Terminal sliding mode control for the trajectory tracking of underactuated autonomous underwater vehicles. Ocean Engineering, 129, 613-625. https://doi.org/10.1016/j.oceaneng.2016.10.032

[24] Perez, T. 2005, Ship Motion Control: Course Keeping and Roll Reduction Using Rudder and Fins, London: Springer.

[25] Healey, A. J., Lienard, D., 1993, Multivariable sliding mode control for autonomous diving and steering of unmanned underwater vehicles. IEEE Journal of Oceanic Engineering. 18(3), 327-339. https://doi.org/10.1109/JOE.1993.236372

[26] Chen, W. H. 2004, Disturbance observer based control for nonlinear systems. IEEE Transactions on Mechatronics, 9(4), 706-710. https://doi.org/10.1109/TMECH.2004.839034

[27] Liu, Z. 2017, Ship adaptive course keeping control with nonlinear disturbance observer. IEEE Access, 5, 17567-17575. https://doi.org/10.1109/ACCESS.2017.2742001

Submitted: $\quad 02.02 .2018$

Accepted: $\quad 08.01 .2019$
Zhiquan Liu

Key Laboratory of Marine Technology and Control Engineering Ministry of Communications, Shanghai Maritime University, Shanghai 201306, China Email: liuzhiquan215@sina.com 